\title{
Analysis of diffraction gratings using an edge element method
}

\author{
Kokou Dossou \\ Department of Mathematical Sciences; University of Technology, Sydney; PO Box 123; \\ Broadway, New South Wales, 200\%; Australia \\ Muthukumaran Packirisamy \\ Department of Mechanical and Industrial Engineering; Concordia University; 1455 de \\ Maisonneuve Boulevard West; Montréal, Québec, H3G 1M8; Canada

\section{Marie Fontaine} \\ Département d'informatique; Université du Québec en Outaouais; Casier Postal 1250, \\ Succursale Hull; Gatineau, Québec, J8X 3X\%; Canada
}

\begin{abstract}
Typically the grating problem is formulated for the TE and TM polarizations, respectively, by using the electric and magnetic fields aligned with the grating wall and perpendicular to the plane of incidence and this leads to a one field component problem. For some grating profiles such as metallic gratings with a triangular profile, the prediction of TM polarization using standard finite element method experiences a slower convergence rate and this reduces the accuracy of the computed results and also introduces a numerical polarization effect. This discrepancy cannot be seen as a simple numerical issue since it has been observed for different types of numerical methods based on the classical formulation. Hence this paper proposes an alternative formulation where the grating problem is modeled by taking the electric field as unknown for TM polarization. The application of this idea to both TE and TM polarizations leads to a two component field problem. The purpose of the present paper is to propose an edge finite element method to solve this wave problem. The comparison between the results of the proposed formulation and the classical formulation shows improvement and robustness by the new approach. (C) 2004 Optical Society of America

OCIS codes: 000.4430, 050.1960
\end{abstract}




\section{Introduction}

For the analysis of the grating properties, either an electric field formulation or a magnetic field formulation can be used for the TE or TM polarizations. However using, for the TE and TM polarizations, respectively the electric and magnetic fields aligned with the grating wall and perpendicular to the plane of incidence is the preferred theory. These formulations lead to a scalar Helmholtz equation involving one electromagnetic field component. For the numerical simulation of periodic non-magnetic media, examples of a slower convergence rate have been observed for some grating profiles of interest to many applications such as finite conductivity metallic gratings with a triangular profile. A slow convergence in numerical prediction requires more refined discretizations to achieve convergence criteria. A significant difference in the convergence rate for the TE and TM polarizations creates an artificial difference in the predicted values resulting in numerical polarization dependence effect which would require even more stringent convergence tolerances to accurately investigate the physical polarization effect.

Convergence problems for the TM polarization have been reported in the literature for

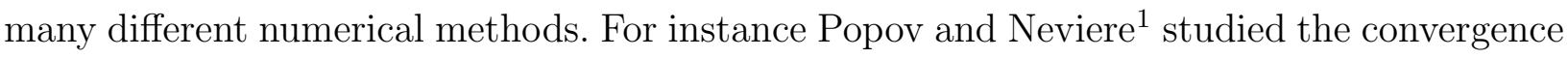
of the differential theory for a wide range of grating profiles and in the particular case of an aluminum triangular profile the TE polarization converges very rapidly in comparison to the TM polarization; as we will see, this problem also occurs when the scalar Helmholtz equation is solved by a finite element method (FEM).

The convergence analysis of the grating problem provides an explanation for this situation ${ }^{2}$. While for the TE case a discontinuous coefficient appears in the lower order terms of the Helmholtz equation, for the TM polarization, it is the second order operator that carries the discontinuous coefficient. Thus the fact that, in the TM case, "the singularities caused by the discontinuous coefficients can spread more destructively" ${ }^{2}$ is a major source of the convergence difficulties.

Thus it appears that the convergence problem cannot be regarded as a simple numerical algorithm issue and it is worth considering the alternative mathematical formulation of the grating problem based on the electric field associated to the TM polarization or the magnetic field associated to the TE polarization. This approach leads to a two-dimensional field problem. To the best of our knowledge, no numerical method based on this alternative formulation has been published. The high computational cost associated with solving twodimensional field problems is a major reason why the focus of most research has relied so far on the classical formulation involving only one field component. The fact that the electric field is no longer continuous across material interfaces can be a problem for some numerical algorithms although vectorial formulations for a wide range of electromagnetic field problems have been solved accurately and efficiently using numerical methods such as the finite element 
methods.

The main goal of the current work is to propose an effective finite element approach for solving the two-dimensional field wave equation. The finite element method is very popular for its flexibility to model complicated geometries with arbitrary material distributions. The standard nodal finite element method can be used ${ }^{3,4}$ to solve grating problems modeled by the scalar Helmholtz equation. It is now widely accepted that edge elements are an effective finite element solver for the double-curl operator in the vectorial equation wave equation. They enforce the continuity of the tangential components and allow the normal component to jump at the element interfaces. Regarding the numerical cost, to evaluate the performance of a finite element method many other factors have to be taken into account in addition to the number of unknowns. For nodal element, the basis functions associated to a vertex is nonzero, on the average, on six neighboring triangles while for edge elements, as we shall see, the basis functions are nonzero only on one triangle or two adjacent triangles so that the matrix systems obtained with edge elements tend to have a lower bandwidth than those from nodal finite elements. Since the computation time of a gaussian elimination scales as $n b^{2}$, where $n$ is the number of unknowns and $b$ the matrix bandwidth, using the 2D field formulation may also be a reasonable and acceptable option.

The finite element method is used to approximate the solution over the modulated grating media and the Rayleigh expansion is used over the homogeneous media. It is well known that such approach introduces a non-local boundary condition along the interface between the homogeneous media and the modulated media. This reduces the FEM matrix sparsity and creates some difficulties in solving by LU factorization. Delâge and Dossou ${ }^{4}$ proposed an efficient approach to solve the non-local transparency condition as they had to solve only Helmholtz equations with local boundary conditions (Dirichlet or Neumann boundary conditions) and a low dimension dense matrix system. This paper adopts such approach to the edge element method. We will consider the cases where the gratings layers are made of metallic and dielectric material. The simplified case of perfect metallic conductor gratings will also be studied.

Here is an outline of the paper. The mathematical modeling of the grating problem using the two-dimensional field variable is presented in section 2. Section 3 is devoted to the finite element discretization of the problem. It is assumed in sections 2 and 3 that the gratings facets are coated with a perfect metallic conductor. Then in section 4 we show how to generalize the models and the numerical method to the case of finite conductivity metallic gratings or dielectric gratings. Presentations and discussions of numerical results follow. 


\section{Mathematical modeling of the grating problem}

\section{A. The wave equation}

The diffraction of light by gratings is analyzed by solving the Maxwell's equations. Depending on whether the $\mathbf{E}$-field or $\mathbf{H}$-field formulation is used, solving these equations in the frequency domain is equivalent to solving the vectorial wave equation

$$
\nabla \times(p \nabla \times \mathbf{v})-k_{0}^{2} q \mathbf{v}=0
$$

where $k_{0}$ is the free space wavenumber, $p=1$ and $q=n^{2}(x, y, z)$ when $\mathbf{v}$ is the electric field $\mathbf{E}$ and $p=1 / n^{2}(x, y, z)$ and $q=1$ when $\mathbf{v}$ is the magnetic field $\mathbf{H} ; n(x, y, z)$ is the refractive index distribution.

It is assumed that a grating has a periodic profile as shown on Fig. 1. The coordinate system is chosen such that the grating layers properties are invariant along the $y$ axis, periodic in the $x$ direction and perpendicular to the $z$ axis. The incident field is usually decomposed into two polarizations: Transverse Electric (TE) $\left(\mathbf{E}=\left(0, E_{y}, 0\right), \mathbf{H}=\left(H_{x}, 0, H_{z}\right)\right)$ and Transverse Magnetic $(\mathrm{TM})\left(\mathbf{E}=\left(E_{x}, 0, E_{z}\right), \mathbf{H}=\left(0, H_{y}, 0\right)\right)$.

The grating modeling based on the fields $\mathbf{v}_{t}=\mathbf{E}_{\mathbf{t}}=\left(E_{x}, E_{z}\right)$ or $\mathbf{v}_{t}=\mathbf{H}_{\mathbf{t}}=\left(H_{x}, H_{z}\right)$ gives a two-dimensional field wave equation involving the double-curl operator:

$$
\nabla_{t} \times\left(p \nabla_{t} \times \mathbf{v}_{t}\right)-k_{0}^{2} q \mathbf{v}_{t}=0
$$

where for an arbitrary two-dimensional field $\mathbf{v}_{t}=\left(v_{x}, v_{z}\right)$ and an arbitrary scalar function $v_{y}$

$$
\nabla_{t} \times \mathbf{v}_{t}=\frac{\partial v_{z}}{\partial x}-\frac{\partial v_{x}}{\partial z} \text { and } \nabla_{t} \times v_{y}=\left[\begin{array}{r}
\frac{\partial v_{y}}{\partial z} \\
-\frac{\partial v_{y}}{\partial x}
\end{array}\right]
$$

\section{B. Boundary condition on the grating facets}

We assume that the grating facets are perfectly conducting metal. The facet profile is described by $z=f(x)$ where $f(x)$ is a periodic function with a period $d$. Due to the periodicity in the $x$ direction, we will only need to solve the diffraction problem over one grating period; this domain is typically decomposed into a homogeneous medium $\Omega^{+}$above the grating tooth and a modulated medium $\Omega$ near the grating tooth as shown in Fig. 1:

$$
\Omega=\{(x, z) \mid 0<x<d, f(x)<z<a\} \text { and } \Omega^{+}=\{(x, z) \mid 0<x<d, a<z\}
$$

where $a$ denotes the maximum value of $f(x)$. For the remaining of this paper we will suppose that the coordinate system are such that $a=0$ as in Fig. 1.

We denote by $\Gamma$ and $\Gamma^{+}$, respectively, the boundaries of $\Omega$ adjacent to the grating facets and the non physical interface between $\Omega$ and $\Omega^{+}$, i.e.,

$$
\Gamma=\{(x, f(x)) \mid 0<x<d\} \text { and } \Gamma^{+}=\{(x, 0) \mid 0<x<d\} .
$$


Let $\mathbf{n}=\left(n_{x}, n_{z}\right)$ represent the outward-pointing normal vector field and $\mathbf{t}=\left(-n_{z}, n_{x}\right)$ be a tangential vector field to the boundaries $\Gamma$ and $\Gamma^{+}$of $\Omega$.

Since the electric field component tangential to the perfectly conducting facets has to be zero, the following boundary conditions on $\Gamma$ are used for the $\mathbf{E}_{t}$-formulation or $\mathbf{H}_{t}$-formulation:

$$
\left\{\begin{array}{l}
\mathbf{v}_{t} \cdot \mathbf{t}=0, \text { if } \mathbf{v}_{t}=\mathbf{E}_{t} \\
\nabla_{t} \times \mathbf{v}_{t}=0, \text { if } \mathbf{v}_{t}=\mathbf{H}_{t}
\end{array}\right.
$$

\section{C. Rayleigh expansion}

Assume that a plane wave

$$
\mathbf{v}_{t}^{i}=\mathbf{R}_{t}^{i} \exp \left(j \alpha_{0} x-j \beta_{0} z\right)
$$

is incident on the echelle grating with an incidence angle $\theta_{0}$; the values of the parameters $\alpha_{0}$ and $\beta_{0}$ and the vector $\mathbf{R}_{t}^{i}$ in the Eq. (7) are

$$
\alpha_{0}=n^{+} k_{0} \sin \theta_{0}, \beta_{0}=n^{+} k_{0} \cos \theta_{0} \text { and } \mathbf{R}_{t}^{i}=\left[\begin{array}{c}
\cos \theta_{0} \\
\sin \theta_{0}
\end{array}\right] .
$$

We shall use the superscript + to refer to the value of a function over $\Omega^{+}$.

From the grating periodicity, it appears that the diffracted field is pseudo-periodic ${ }^{5}$, i.e., a product of $\exp \left(j \alpha_{0} x\right)$ by a field $\mathbf{v}_{t}^{d}(x, z)$ which is $d$-periodic with respect to the variable $x$. So the solution is fully determined by solving the wave problem over one grating period.

Now let $\mathbf{v}_{t}^{d}$ represents the diffracted field and $\mathbf{v}_{t}$ the total field, i.e., $\mathbf{v}_{t}=\mathbf{v}_{t}^{i}+\mathbf{v}_{t}^{d}$. The diffracted field admits the following Rayleigh expansion over $\Omega^{+}$

$$
\mathbf{v}_{t}^{d}=\sum_{m=-\infty}^{+\infty}\left[\begin{array}{c}
A_{m}^{+} \\
B_{m}^{+}
\end{array}\right] \exp \left(j \alpha_{m} x+j \beta_{m}^{+} z\right)
$$

where

$$
\alpha_{m}=\alpha+m \frac{2 \pi}{d} \text { and } \beta_{m}^{+}=\left\{\begin{array}{l}
\sqrt{\left(n^{+}\right)^{2} k_{0}^{2}-\alpha_{m}^{2}}, \text { if }\left(n^{+}\right)^{2} k_{0}^{2}-\alpha_{m}^{2} \geq 0 \\
j \sqrt{\alpha_{m}^{2}-\left(n^{+}\right)^{2} k_{0}^{2}}, \text { if }\left(n^{+}\right)^{2} k_{0}^{2}-\alpha_{m}^{2}<0 .
\end{array}\right.
$$

Note that the coefficients $\beta_{m}^{+}$are chosen in such a way that the radiation condition ${ }^{5}$ is satisfied. When $\beta_{m}^{+}$is an imaginary complex number, the corresponding diffraction order is an evanescent wave and does not carry power away from the grating. A propagating diffraction order corresponds to a real number value of $\beta_{m}^{+}$. From the divergence free condition, the Rayleigh coefficients $A_{m}$ and $B_{m}$ satisfy the following relation

$$
\alpha_{m} A_{m}^{+}+\beta_{m}^{+} B_{m}^{+}=0
$$

so that the Rayleigh expansion can be written in the form

$$
\mathbf{v}_{t}^{d}=\sum_{m=-\infty}^{+\infty} A_{m}^{+} \mathbf{R}_{t}^{m+} \exp \left(j \alpha_{m} x+j \beta_{m}^{+} z\right)
$$


with

$$
\mathbf{R}_{t}^{m+}=\frac{1}{n^{+} k_{0}}\left[\begin{array}{c}
-\beta_{m}^{+} \\
\alpha_{m}
\end{array}\right] .
$$

The diffraction efficiency of the $m^{\text {th }}$ propagating diffraction order is given by

$$
e_{m}^{+}=A_{m}^{+}\left(A_{m}^{+}\right)^{*} \frac{\beta_{m}^{+}}{\beta_{0}}
$$

where $^{*}$ denotes the complex conjugate operator.

\section{D. Transmission condition}

A transparency condition is enforced across the artificial boundary $\Gamma^{+}$by imposing the continuity of the tangential component of the electric and magnetic fields. For the present problem this means that the Rayleigh coefficients $A_{m}^{+}$have to be such that $\mathbf{v}_{t} \cdot \mathbf{t}$ and $\left(p \nabla_{t} \times \mathbf{v}_{t}\right)$ are continuous across $\Gamma^{+}$:

$$
\left\{\begin{array}{l}
\mathbf{v}_{t} \cdot \mathbf{t}=\left(\mathbf{R}_{t}^{i} \cdot \mathbf{t}\right) \exp \left(j \alpha_{0} x\right)+\sum_{m=-\infty}^{+\infty} A_{m}^{+} \exp \left(j \alpha_{m} x\right)\left(\mathbf{R}_{t}^{m+} \cdot \mathbf{t}\right), \\
\left(p \nabla_{t} \times \mathbf{v}_{t}\right)=j n^{+} k_{0} p^{+} \exp \left(j \alpha_{0} x\right)+j n^{+} k_{0} p^{+} \sum_{m=-\infty}^{+\infty} A_{m}^{+} \exp \left(j \alpha_{m} x\right) .
\end{array}\right.
$$

For the Eq. $(15)_{2}$, the following relation is used:

$$
\nabla_{t} \times\left(\mathbf{R}_{t}^{m+} \exp \left(j \alpha_{m} x+j \beta_{m}^{+} z\right)\right)=n^{+} k_{0} \exp \left(j \alpha_{m} x+j \beta_{m}^{+} z\right) .
$$

Then, from Eqs. (2), (6) and (15) and since $\mathbf{t}=(-1,0)$ on $\Gamma^{+}$, the grating diffraction problem can be formulated as:

Find the field $\mathbf{v}_{t}$ and the Rayleigh coefficients $A_{m}^{+}$such that

$$
\left\{\begin{array}{l}
\nabla_{t} \times\left(p \nabla_{t} \times \mathbf{v}_{t}\right)-k_{0}^{2} q \mathbf{v}_{t}=0, \text { in } \Omega, \\
\mathbf{v}_{t} \cdot \mathbf{t}=0, \text { on } \Gamma, \text { if } \mathbf{v}_{t}=\mathbf{E}_{t},\left(\text { or } \nabla_{t} \times \mathbf{v}_{t}=0, \text { on } \Gamma, \text { if } \mathbf{v}_{t}=\mathbf{H}_{t}\right), \\
-v_{x}=\frac{1}{n^{+} k_{0}}\left(-\beta_{0} \exp \left(j \alpha_{0} x\right)+\sum_{m=-\infty}^{+\infty} \beta_{m}^{+} A_{m}^{+} \exp \left(j \alpha_{m} x\right)\right), \text { on } \Gamma^{+}, \\
\left(p \nabla_{t} \times \mathbf{v}_{t}\right)=\left(j n^{+} k_{0} p^{+}\right)\left(\exp \left(j \alpha_{0} x\right)+\sum_{m=-\infty}^{+\infty} A_{m}^{+} \exp \left(j \alpha_{m} x\right)\right), \text { on } \Gamma^{+} .
\end{array}\right.
$$

\section{Variational formulation and finite element solution}

\section{A. Variational formulation}

Over the domain $\Omega$, the field solution is approximated by an FEM solution. When the FEM is used to solve partial differential equations, the equations must be rewritten in an equivalent 
variational formulation before being discretized. Taking the dot product of Eq. (17) $)_{1}$ with a test function $\mathbf{w}_{t}=\left[w_{x}, w_{z}\right]^{T}$ and integrating by parts over $\Omega$, leads to the equation:

$$
\int_{\Omega}\left(p\left(\nabla_{t} \times \mathbf{v}_{t}\right)\left(\nabla_{t} \times \mathbf{w}_{t}^{*}\right)-k_{0}^{2} q \mathbf{v}_{t} \cdot \mathbf{w}_{t}^{*}\right) \mathrm{d} x \mathrm{~d} z-\int_{\Gamma \cup \Gamma^{+}} p\left(\nabla_{t} \times \mathbf{v}_{t}\right)\left(\mathbf{w}_{t}^{*} \cdot \mathbf{t}\right) \mathrm{d} s=0 .
$$

If $\mathbf{v}_{t}=\mathbf{E}_{t}$, the corresponding boundary condition in Eq. $(17)_{2}$, i.e., $\mathbf{v}_{t} \cdot \mathbf{t}=0$ on $\Gamma$, is also imposed on the test function $\mathbf{w}_{t}$. However for the case $\mathbf{v}_{t}=\mathbf{H}_{t}$, no boundary condition is enforced on the test function $\mathbf{w}_{t}$, because the condition $\nabla_{t} \times \mathbf{v}_{t}=0$ on $\Gamma$ is a natural boundary condition to the variational formulation.

From the boundary condition $(17)_{2}$, it follows that the line integral along $\Gamma$ cancels while from Eq. $(17)_{4}$ we have:

$$
\begin{aligned}
& \int_{\Gamma^{+}} p\left(\nabla_{t} \times \mathbf{v}_{t}\right)\left(\mathbf{w}_{t}^{*} \cdot \mathbf{t}\right) \mathrm{d} s=j n^{+} k_{0} p^{+} \int_{0}^{d} \exp \left(j \alpha_{0} x\right)\left(-w_{x}^{*}\right) \mathrm{d} x \\
& +j n^{+} k_{0} p^{+} \sum_{m=-\infty}^{+\infty} A_{m}^{+} \int_{0}^{d} \exp \left(j \alpha_{m} x\right)\left(-w_{x}^{*}\right) \mathrm{d} x .
\end{aligned}
$$

So that

$$
\begin{aligned}
& \int_{\Omega}\left(p\left(\nabla_{t} \times \mathbf{v}_{t}\right)\left(\nabla_{t} \times \mathbf{w}_{t}^{*}\right)-k_{0}^{2} q \mathbf{v}_{t} \cdot \mathbf{w}_{t}^{*}\right) \mathrm{d} x \mathrm{~d} z \\
& +j n^{+} k_{0} p^{+} \sum_{m=-\infty}^{+\infty} A_{m}^{+} \int_{0}^{d} \exp \left(j \alpha_{m} x\right) w_{x}^{*} \mathrm{~d} x \\
& =-\left(j n^{+} k_{0} p^{+}\right) \int_{0}^{d} \exp \left(j \alpha_{0} x\right) w_{x}^{*} \mathrm{~d} x .
\end{aligned}
$$

Taking the inner product of Eq. (17) $)_{3}$ with $\exp \left(j \alpha_{m} x\right)$ and using the orthogonality of the exponential functions $\exp \left(j \alpha_{m} x\right)$ lead to

$$
\frac{1}{n^{+} k_{0}} \beta_{m}^{+} A_{m}^{+} d+\int_{0}^{d} v_{x}(x, 0) \exp \left(-j \alpha_{m} x\right) \mathrm{d} x=\frac{1}{n^{+} k_{0}} \beta_{0} d \delta_{m 0},
$$

where $\delta_{m s}$ stands for the Kronecker symbol, i.e., $\delta_{m s}=1$ if $m=s$ and $\delta_{m s}=0$ otherwise.

Finally the variational problem can be formulated as:

Find the field $\mathbf{v}_{t}$ and the Rayleigh coefficients such that Eq. (20) is true for all test functions $\mathbf{w}_{t}$ and $E q$. (21) is true for all integers $m$.

\section{B. Finite element discretization}

The discretization of Eqs. (20) and (21) is performed by using a vector edge finite element. Edge elements were introduced by Nédélec ${ }^{6,7}$ and more details about the type of edge elements used in this paper can be found in other publications ${ }^{8-10}$. Edge elements are well known for their ability to efficiently approximate the curl-curl operator. We use quadratic edge elements to approximate the field $\mathbf{v}_{t}$. In each FEM element $e$ the field $\mathbf{v}_{t}$ is expanded as

$$
\mathbf{v}_{t}=\sum_{i=1}^{12} u_{i} \mathbf{v}_{i} .
$$


The polynomial expression of the edge vector basis functions for the quadratic elements are explained in Table 1 . The scalar $u_{i}, i=1, \ldots, 12$, are the FEM solution components associated to this edge basis.

The technique used for the basis construction is also valid for curved elements and is relatively simple, especially for higher order elements. For the case of quadratic elements, in each FEM element, the shape functions $\mathbf{v}_{8}, \mathbf{v}_{10}$ and $\mathbf{v}_{12}$ have a zero tangential component on all three triangle edges so there is no inter-element tangential continuity to be enforced and these functions can be considered as attached to the interior of the triangle; each of the other shape functions has a nonzero tangential component only on one triangle edge.

To justify our affirmations, let us consider, for instance, the basis function $\mathbf{v}_{1}=L_{1}^{2}\left(\nabla_{t} L_{2}^{1}\right)$ (the other cases can be treated in a similar way). The tangential component of $\mathbf{v}_{1}$ is zero on the edge $e_{1}$ (the edge opposed to vertex 1) because $L_{1}^{2}=0$ on that edge. Since $L_{2}^{1}=0$ on the edge $e_{2}$ (the edge opposed to vertex 2), $\nabla_{t} L_{2}^{1}$ is perpendicular to $e_{2}$, so the tangential component of $\mathbf{v}_{1}$ is also zero on $e_{2}$. Thus we only have to enforce the continuity of the tangential component across the edge $e_{3}$ if it belongs to two adjacent triangles. In the context of the finite element method, the continuity across $e_{3}$ of the scalar Lagrange function $L_{1}^{2}$ attached to the vertex 1 can be easily realized, so that we will only discuss the tangential continuity of the gradient $\left(\nabla_{t} L_{2}^{1}\right)$. The tangential component of $\left(\nabla_{t} L_{2}^{1}\right)$ on $e_{3}$ is equal to the tangential derivative of $L_{2}^{1}$ along $e_{3}$. Since $L_{2}^{1}$ is continuous across $e_{3}$ so is its tangential derivative. Thus $\mathbf{v}_{1}=L_{1}^{2}\left(\nabla_{t} L_{2}^{1}\right)$ is tangentially continuous across $e_{3}$.

To obtain the numerical solutions we truncate the Rayleigh expansion to a finite number $\left(P_{2}-P_{1}+1\right)$ of orders varying from $P_{1}$ to $P_{2}$ where the integer parameters $P_{1}$ and $P_{2}$ are usually chosen such that the truncated Rayleigh expansion includes all the propagating orders and some of the evanescent diffraction orders. $P_{1}$ and $P_{2}$ will be called the truncation parameters and since the propagating orders are not generally distributed symmetrically around the zero order, we can have $P_{1} \neq-P 2$.

The letter $D$ will refer to the dimension of the FEM space. The vectors $\{U\}$ and $\{A\}$ will respectively designate the vector of FEM basis components and the vector of Rayleigh coefficients. Application of Galerkin procedure to Eqs. (20) and (21) leads to the following linear system

$$
\left[\begin{array}{ll}
M_{U U} & M_{U A} \\
M_{A U} & M_{A A}
\end{array}\right]\left[\begin{array}{c}
U \\
A
\end{array}\right]=\left[\begin{array}{l}
F_{U} \\
F_{A}
\end{array}\right]
$$


where the matrix of the system is defined as

$$
\begin{aligned}
& \left(M_{U U}\right)_{m s}=\int_{\Omega}\left(p\left(\nabla_{t} \times \mathbf{v}_{s}\right)\left(\nabla_{t} \times \mathbf{v}_{m}^{*}\right)-k_{0}^{2} q \mathbf{v}_{s} \cdot \mathbf{v}_{m}^{*}\right) \mathrm{d} x \mathrm{~d} z, m, s=1, \ldots, D \\
& \left(M_{U A}\right)_{m s}=j n^{+} k_{0} p^{+} \int_{0}^{d} v_{m x}^{*}(x, 0) \exp \left(j \alpha_{s+P_{1}-1} x\right) \mathrm{d} x \\
& m=1, \ldots, D, s=1, \ldots,\left(P_{2}-P_{1}+1\right) \\
& \left(M_{A U}\right)_{m s}=\int_{0}^{d} v_{s x}(x, 0) \exp \left(-j \alpha_{m+P_{1}-1} x\right) \mathrm{d} x \\
& m=1, \ldots,\left(P_{2}-P_{1}+1\right), s=1, \ldots, D \\
& \left(M_{A A}\right)_{m s}=\frac{\beta_{m+P_{1}-1}^{+} d}{n^{+} k_{0}} \delta_{m s}, \quad m, s=1, \ldots,\left(P_{2}-P_{1}+1\right)
\end{aligned}
$$

For the coefficients $\left(M_{U U}\right)_{m s}$, the computation of second order derivatives can be avoided by using of the relation $\nabla_{t} \times\left(f \nabla_{t} g\right)=\operatorname{det}\left[\nabla_{t} f, \nabla_{t} g\right]$.

The coefficients of the source terms are

$$
\begin{aligned}
& \left(F_{U}\right)_{m}=-j n^{+} k_{0} p^{+} \int_{0}^{d} v_{m x}^{*} \exp \left(j \alpha_{0} x\right) \mathrm{d} x, m=1, \ldots, D, \\
& \left(F_{A}\right)_{m}=\frac{\beta_{0} d}{n^{+} k_{0}} \delta_{\left(m+P_{1}-1\right) 0}, m=1, \ldots,\left(P_{2}-P_{1}+1\right) .
\end{aligned}
$$

The sub-matrix $\left[M_{U U}\right]$ is a standard finite element matrix and has a sparse band matrix profile. However, since the Rayleigh functions $\exp \left(j \alpha_{m} x\right)$ vary along the boundary $\Gamma^{+}$, the columns of $\left[M_{U A}\right]$ and the rows of $\left[M_{A U}\right]$ have in general nonzero coefficients for the degrees of freedom corresponding to FEM nodes located on $\Gamma^{+}$. Hence, the bandwidth of the full system matrix in Eq. (23) can be quite large. This is an unfavorable situation as many existing finite element tools are optimized for sparse and relatively small bandwidth matrices. Described here an approach to efficiently solve the Eq. (23) by using standard finite element packages. This approach has been successively applied to the scalar Helmholtz problem ${ }^{4}$.

First express $\{U\}$ in term of $\{A\}$

$$
\{U\}=\left[M_{U U}\right]^{-1}\left\{F_{U}\right\}-\left[M_{U U}\right]^{-1}\left[M_{U A}\right]\{A\},
$$

then substitution into Eq. $(23)_{2}$ leads to the following low dimension and dense matrix system

$$
\left[\hat{M}_{A A}\right]\{A\}=\left\{\hat{F}_{A}\right\}
$$

where the matrix $\left[\hat{M}_{A A}\right]$ and the vector $\left\{\hat{F}_{A}\right\}$ are given by

$$
\left[\hat{M}_{A A}\right]=\left[M_{A A}\right]-\left[M_{A U}\right]\left[M_{U U}\right]^{-1}\left[M_{U A}\right]
$$

and

$$
\left\{\hat{F}_{A}\right\}=\left\{F_{A}\right\}-\left[M_{A U}\right]\left[M_{U U}\right]^{-1}\left\{F_{U}\right\}
$$


We now describe a method to construct the matrix $\left[\hat{M}_{A A}\right]$ and the vector $\left\{\hat{F}_{A}\right\}$. The vector $\left[M_{U U}\right]^{-1}\left\{F_{U}\right\}$ and the columns of the matrix $\left[M_{U U}\right]^{-1}\left[M_{U A}\right]$ can be computed by solving the linear systems

$$
\left[M_{U U}\right]\left\{\hat{U}^{i}\right\}=\left\{F_{U}\right\} \text { and }\left[M_{U U}\right]\left\{\hat{U}_{m}\right\}=\left\{\hat{F}_{m}\right\},
$$

where for $m=1, \ldots,\left(P_{2}-P_{1}+1\right),\left\{\hat{F}_{m}\right\}$ is the $m^{\text {th }}$ column of the sub-matrix $\left[M_{U A}\right]$. The multiple resolutions of the linear systems (30) can be carried out efficiently by using LU factorization.

After $\left\{\hat{U}^{i}\right\}$ and $\left\{\hat{U}_{m}\right\}$, for $m=1, \ldots,\left(P_{2}-P_{1}+1\right)$, are computed, $\left[\hat{M}_{A A}\right]$ and $\left\{\hat{F}_{A}\right\}$ can be obtained as:

$$
\begin{gathered}
\left(\hat{M}_{A A}\right)_{m s}=\left(M_{A A}\right)_{m s}-\int_{0}^{d} \hat{U}_{s x}(x, 0) \exp \left(-j \alpha_{m+P_{1}-1} x\right) \mathrm{d} x \\
m, s=1, \ldots,\left(P_{2}-P_{1}+1\right) \\
\left(\hat{F}_{A}\right)_{m}=\left(F_{A}\right)_{m}-\int_{0}^{d} \hat{U}_{x}^{i}(x, 0) \exp \left(-j \alpha_{m+P_{1}-1} x\right) \mathrm{d} x \\
m=1, \ldots,\left(P_{2}-P_{1}+1\right) .
\end{gathered}
$$

Once the system (27) is solved, from the Eq. (26), the field $\{U\}$ is given by

$$
\{U\}=\left\{\hat{U}^{i}\right\}-\sum_{m=1}^{P_{2}-P_{1}+1} A_{m}^{+}\left\{\hat{U}_{m}\right\} .
$$

It is worth noting that Eq. (32) corresponds to a discretized and truncated form of the following expansion which can be formally obtained from the Eq. (20) as

$$
\mathbf{v}_{t}=\mathbf{u}_{t}^{i}+\sum_{m=-\infty}^{+\infty} A_{m}^{+} \mathbf{u}_{t m}
$$

where $\mathbf{u}_{t}^{i}$ and $\mathbf{u}_{t m}$ are defined similarly to $\left\{\hat{U}^{i}\right\}$ and $\left\{\hat{U}_{m}\right\}$ as solutions to the continuous problems associated to the discretized systems (30).

\section{The case of metallic or dielectric gratings}

In the general case of metallic or dielectric gratings, the transmitted wave propagating in the lower media has to be taken into account in addition to the reflected wave propagating in the upper media. Figure 3 presents some of the notations that will be used in this section. The domain $\Omega$ is one elementary cell of the periodic inhomogeneous media; $\Omega^{+}$and $\Omega^{-}$are respectively the upper and lower homogeneous media.

\section{A. Pseudo-periodic boundary conditions}

Pseudo-periodic boundary conditions are set on the boundaries $\Gamma^{L}$ and $\Gamma^{R}$ in Fig. 3. Let $P^{L}=\left(x^{L}, z^{L}\right)$ be a point lying on $\Gamma^{L}$ and $P^{R}=\left(x^{L}+d, z^{L}\right)$ the corresponding point of $\Gamma^{R}$ 
that is one period away from $P^{L}$. Then at $P^{L}$ and $P^{R}$, the tangential components of the electric and magnetic fields satisfy the following pseudo-periodic boundary conditions:

$$
\left\{\begin{array}{l}
\mathbf{E}\left(P^{R}\right) \cdot \mathbf{z}=\exp (j \alpha d)\left(\mathbf{E}\left(P^{L}\right) \cdot \mathbf{z}\right) \\
\mathbf{H}\left(P^{R}\right) \cdot \mathbf{z}=\exp (j \alpha d)\left(\mathbf{H}\left(P^{L}\right) \cdot \mathbf{z}\right),
\end{array}\right.
$$

where $\mathbf{z}=(0,1)$ is a tangent vector to $\Gamma^{L}$ and $\Gamma^{R}$.

For the $\mathbf{v}_{t}$ formulation, the conditions (34) become

$$
\left\{\begin{array}{l}
\mathbf{v}_{t}\left(P^{R}\right) \cdot \mathbf{z}=\exp (j \alpha d)\left(\mathbf{v}_{t}\left(P^{L}\right) \cdot \mathbf{z}\right) \\
\left(p \nabla \times \mathbf{v}_{t}\right)\left(P^{R}\right)=\exp (j \alpha d)\left(p \nabla \times \mathbf{v}_{t}\right)\left(P^{L}\right) .
\end{array}\right.
$$

\section{B. Rayleigh expansion}

The diffracted field propagating in $\Omega^{+}$is represented by the Rayleigh expansion given by the Eq. (12). The wave propagating in $\Omega^{-}$can be expressed as

$$
\mathbf{v}_{t}^{d}=\sum_{m=-\infty}^{+\infty} A_{m}^{-} \mathbf{R}_{t}^{m-} \exp \left(j \alpha_{m} x-j \beta_{m}^{-}(z+h)\right),
$$

where $h$ denotes the thickness of $\Omega$, i.e., the distance between the boundaries $\Gamma^{+}$and $\Gamma^{-}$,

$$
\alpha_{m}=\alpha+m \frac{2 \pi}{d} \text { and } \beta_{m}^{-}=\left\{\begin{array}{l}
\sqrt{\left(n^{-}\right)^{2} k_{0}^{2}-\alpha_{m}^{2}}, \text { if }\left(n^{-}\right)^{2} k_{0}^{2}-\alpha_{m}^{2} \geq 0 \\
j \sqrt{\alpha_{m}^{2}-\left(n^{-}\right)^{2} k_{0}^{2}}, \text { if }\left(n^{-}\right)^{2} k_{0}^{2}-\alpha_{m}^{2}<0
\end{array}\right.
$$

and

$$
\mathbf{R}_{t}^{m-}=\frac{1}{n^{-} k_{0}}\left[\begin{array}{c}
\beta_{m}^{-} \\
\alpha_{m}
\end{array}\right]
$$

The diffraction efficiency of the $m^{\text {th }}$ diffraction order propagating in $\Omega^{-}$is given by

$$
e_{m}^{-}=A_{m}^{-}\left(A_{m}^{-}\right)^{*} \frac{p^{-} \beta_{m}^{-}}{p^{+} \beta}
$$

\section{C. Transmission condition}

Over the boundary $\Gamma^{+}$the transmission conditions provided by the Eq. (15) still apply. The adaptation of these boundary conditions to the lower boundary $\Gamma^{-}$is straightforward.

4.D. Variational formulation and finite element solution

From the wave equation (2) and the boundary conditions on $\Gamma^{+}, \Gamma^{-}, \Gamma^{L}$ and $\Gamma^{L}$ we have the following variational problem: 
Find the field $\mathbf{v}_{t}$ and the Rayleigh coefficients such that for all test functions $\mathbf{w}_{t}$

$$
\begin{aligned}
\int_{\Omega}( & \left.p\left(\nabla_{t} \times \mathbf{v}_{t}\right)\left(\nabla_{t} \times \mathbf{w}_{t}^{*}\right)-k_{0}^{2} q \mathbf{v}_{t} \cdot \mathbf{w}_{t}^{*}\right) \mathrm{d} x \mathrm{~d} z \\
& +\left(j n^{+} k_{0} p^{+}\right) \sum_{m=-\infty}^{+\infty} A_{m}^{+} \int_{0}^{d} \exp \left(j \alpha_{m} x\right) w_{x}^{*}(x, 0) \mathrm{d} x \\
& -\left(j n^{-} k_{0} p^{-}\right) \sum_{m=-\infty}^{+\infty} A_{m}^{-} \int_{0}^{d} \exp \left(j \alpha_{m} x\right) w_{x}^{*}(x,-h) \mathrm{d} x \\
= & -\left(j n^{+} k_{0} p^{+}\right) \int_{0}^{d} \exp \left(j \alpha_{0} x\right) w_{x}^{*}(x, 0) \mathrm{d} x
\end{aligned}
$$

and for all integers $m$

$$
\left\{\begin{array}{l}
\frac{\beta_{m}^{+} d}{n^{+} k_{0}} A_{m}^{+}+\int_{0}^{d} v_{x}(x, 0) \exp \left(-j \alpha_{m} x\right) \mathrm{d} x=\frac{\beta_{0} d}{n^{+} k_{0}} \delta_{m 0} \\
\frac{\beta_{m}^{-} d}{n^{-} k_{0}} A_{m}^{-}-\int_{0}^{d} v_{x}(x,-h) \exp \left(-j \alpha_{m} x\right) \mathrm{d} x=0
\end{array}\right.
$$

The finite element discretization of Eqs. (40) and (41) leads to a linear system of equations similar to Eq. (23). Note that only the first pseudo-periodicity condition $(35)_{1}$ is explicitly enforced on the of the finite element basis functions which have a nonzero tangential components on $\Gamma^{L}$ and $\Gamma^{R}$. The other pseudo-periodicity condition $(35)_{2}$ is satisfied by the solution through the natural boundary condition in the variational process. The solution construction approach used in section 3 can be also applied here. However, since in Eq. (35) the point $P^{R}$ is not in the immediate vicinity of $P^{L}$, the pseudo-periodicity condition is a non-local boundary condition thus, in the same way as we did for the resolution of the linear system (23), a block resolution method can be used for the step corresponding to the linear systems (30).

\section{Numerical results}

\section{A. Comparison with other methods}

In order to compare the finite element methods with other numerical techniques, let us consider, for instance, a triangular grating already analyzed by Li et al. ${ }^{11}$ using the $\mathrm{C}$ method and the Fourier modal method (FMM). Here are the parameters of the grating problem: grating apex angle $90^{\circ}$, left hand base angle $30^{\circ}, n^{+}=1, n^{-}=1+5 j, d=2 \lambda$, and angle of incidence $\theta_{0}=15^{\circ} ; \lambda$ is the free space wavelength of the incident light.

Let us recall that $v_{y}$ refers to the conventional scalar field formulation while $\mathbf{v}_{t}$ refers to the present proposed formulation. The results presented in Table 2 show a good agreement between the $\mathrm{C}$ method and the two FEM based on the field $v_{y}$ or $\mathbf{v}_{t}$. However, for the TM case, the results of the FMM method are significantly different from those of the other methods. The Table 3 shows that the two types of FEM have a good convergence behavior for this low order diffraction problem. 
For the remainder of this section, we will compare the convergence rate of the $v_{y}$ and $\mathbf{v}_{t}$ formulations for various echelle grating profiles.

\section{B. Grating parameters}

The grating parameters used in this section are motivated by the work in ${ }^{4,12}$ where planar waveguide echelle grating based demultiplexers for optical communication were discussed. For an optimized demultiplexer design, a suitable selection of grating parameters is important and it is determined by design specifications such as the linear dispersion, the wavelength region of operation and the device size ${ }^{13}$. Typically demultiplexers based on planar waveguide gratings operate in the $1.55 \mu \mathrm{m}$ wavelength region. Papers describing integrated echelle grating demultiplexers have been published by many groups. For instance, Clemens et al. ${ }^{14}$ realized a planar 8 -channel optical demultiplexer whose grating component is blazed at $55^{\circ}$ and is operated in the 30 th and 50 th diffraction order. He et al. ${ }^{15}$ developed a 10 -channel wavelength demultiplexers using grating blazed at $54.5^{\circ}$ and operated in the 12 th order. More recently, Janz et al. ${ }^{12}$ presented demultiplexers with 48 channels and 256 channels based on an integrated planar grating operating in the 20th order.

In order to simplify the analysis, the planar waveguide is represented by a homogeneous medium whose refractive index is set to the waveguide mode effective index and an infinite and periodic grating is assumed. Let us, for example, consider a triangular profile grating (echelle grating) as shown in Figs. 1 and 3 with $55^{\circ}$ blaze angle and $90^{\circ}$ apex angle. The grating surface is illuminated by light with incidence angle $\theta_{0}=56^{\circ}$. The refractive indices of the homogeneous grating media are $n^{+}=1.45$ and $n^{-}=1.0$. The refractive index of the metallic coating is $n=1.47+15.75 j$ (aluminum) and its thickness is $4 \mu \mathrm{m}$.

The operating order of the grating is $N_{0}=-22$ with a period $d$ chosen such that the Littrow configuration is realized for the wavelength $\lambda=1.55 \mu \mathrm{m}$, the diffraction order of $N_{0}=-22$ and the incidence angle of $\theta=55^{\circ}$, i.e., $d=-N_{0} \lambda /\left(2 n^{+} \sin \theta\right)$.

As the planar waveguide based gratings with order of around 20 and blaze angle of around $55^{\circ}$ are realizable and useful for telecommunication applications, this paper aims at studying gratings with such parameters. Even though the proposed method can be used for a wide range of grating applications, telecommunication application is chosen only for demonstration purpose.

\section{C. Polarization dependent loss and its origin}

Metal coated echelle grating are well known to exhibit an important dependence of the diffraction efficiency on the polarization state and this dependence is sometimes called polarization dependent loss (PDL) and is measured by the difference of the efficiencies (in $\mathrm{dB}$ ) of the TE and TM polarizations. The main source of the PDL is the different polarization 
behavior at metal-dielectric interface ${ }^{16}$. At a metal-dielectric interface, the tangential component of the electric field is always zero for a perfect conductor and remains small for metal with finite conductivity. To simplify the modeling, the perfect conductor metal-dielectric interface is assumed. There are two types of metallic facets, namely, the reflecting facets that face the incident light and the non-reflecting facets that are aligned with the light. For TE polarization, the incident electric field $\mathbf{E}=\left(0, E_{y}, 0\right)$ is tangential to both reflecting and non-reflecting facets so that the total electric field is zero on all the grating facets. For TM polarization, the incident electric field $\mathbf{E}=\left(E_{x}, 0, E_{z}\right)$ is within a few degrees tangential to the reflecting facets and normal to the non-reflecting facets. Hence there is a net difference in the boundary conditions for the TE and TM polarizations on the non-reflecting facets and that is a major source of the grating PDL. For telecommunication applications the requirement for low PDL is critical. In order to reduce the PDL, it is natural to consider removing the metallic coating from the non-reflecting facets and to have a dielectric-dielectric interface, instead.

Presented are the numerical simulation results that confirm the validity of this approach. We will consider the following cases:

1. All the grating facets are coated with a perfect metallic conductor layer

2. Only the reflecting facets are coated with a perfect metallic conductor layer

3. All the grating facets are coated with a finite conductivity metal

4. Only the reflecting facets are coated with a finite conductivity metal

\section{D. Grating with perfect metallic conductor on both types of facets}

The diffraction efficiencies were computed using the same FEM meshes and truncation parameters for the $v_{y}$ and $\mathbf{v}_{t}$ formulations. Figure 4 shows a good agreement between the efficiencies (diffraction order -22) and the PDL obtained with both $v_{y}$ and $\mathbf{v}_{t}$ formulations. The PDL over the wavelength range considered is $5 \mathrm{~dB}$. The results on Fig. 4 were obtained on a quadratic FEM mesh having 9970 triangles and 20283 nodes and the truncation parameters are -36 and 14 . At each wavelength, the computing time for the $v_{y}$ and $\mathbf{v}_{t}$ formulations are respectively about 4 seconds and 31 seconds (on a Pentium 4 computer).

\section{E. Perfect metallic conductor on reflecting facets: Low PDL grating}

The grating has the same profile as in sub-section 5.D except the fact that metal is removed from the non-reflecting facets. In order to take into account the transmitted light, the Rayleigh expansion is used both above and below the grating. The FEM mesh used for the computation has 5018 triangles and 10261 nodes. The truncation parameters are -36 
and 14. To obtain the solution at a given wavelength, it takes around 8 seconds for the $v_{y}$ method and 46 seconds for the $\mathbf{v}_{t}$ method. Figure 5 presents the computed results for the operating order -22 . It can be observed that the peak efficiency of the TM polarization shifts to a lower wavelength near the TE polarization maximum efficiency and this shift results in a dramatic reduction of the PDL values. Note that this low PDL effect has been confirmed by experimental measurements ${ }^{12}$.

\section{F. Grating facet coated with metal of finite conductivity}

The computed numerical results are presented in Fig. 6. As in the case of grating with perfectly conductive facets, the PDL is high and is around 6dB. An FEM mesh containing 10919 triangles and 22122 nodes is used and the truncation parameters are -36 and 14. The CPU time spent solving each problem is approximately 23 seconds for the $v_{y}$ formulation and 151 seconds for the $\mathbf{v}_{t}$ formulation. The efficiency curves of the TE polarization computed with the $E_{y}$ and $\mathbf{H}_{t}$ formulations are very close. However there is a significant difference for the TM efficiency curves obtained with the $H_{y}$ and $\mathbf{E}_{t}$ formulations. It can be attributed to the poor convergence of the $H_{y}$ formulation as seen from the convergence curves in Fig. 7 where the solutions are computed on four increasingly refined meshes whose parameters are given in Table 4. In particular, compared with the other methods, the convergence of the $H_{y}$ method to the maximum diffraction efficiency is too slow. Because of the limited memory space, the computation on the mesh (4) in Table 4, with more than 30000 triangles, is done only for the $E_{y}$ and $H_{y}$ methods. Figure 8 shows the convergence of the computed PDL. The PDL obtained with the $\mathbf{v}_{t}$ method converges very quickly in comparison with the $v_{y}$ method. This can be explained by the fact that the net difference in the convergence rates of the $E_{y}$ and $H_{y}$ formulation creates a numerical polarization effect that pollutes the physical polarization dependence. Since the $\mathbf{E}_{t}$ and $\mathbf{H}_{t}$ formulations have a comparable convergence rate, the impact of a numerical polarization effect is less significant.

\section{G. Grating with a finite conductivity metal on reflecting facets only: Low PDL grating}

The same data as in sub-section 5.F is considered except the fact that metal is removed from the non-reflecting facets. The curves on Fig. 9 are closely related to those obtained for the low PDL perfect metallic conductor grating on Fig. 5.

\section{Conclusion}

The formulation of grating problems based on the electromagnetic field components $\left(H_{x}, H_{z}\right)$ and $\left(E_{x}, E_{z}\right)$ for, respectively, the TE and TM polarizations can be accurately and efficiently solved by using an edge finite element method. This approach gives the field components that are not readily available with the classic $E_{y}$ and $H_{y}$ based formulations which, for the 
sake of comparison, are also solved using a finite element technique. For the grating problems analyzed in this paper, the $\mathbf{v}_{t}$ formulations have consistently behave well for both polarizations however it takes a longer computation time than the traditional approach except in the case of finite conductivity metallic grating in the TM polarization where the $H_{y}$ formulations experiences a slow convergence and requires more computation resource to achieve a comparable level of accuracy as the new method. Thus considering the overall performance, the new method appears to be more reliable. However none of these two different approaches compares favorably with the other for every grating problem so researchers should consider both approaches.

Finally the good convergence behavior of the $\mathbf{H}_{t}$ field formulation is somewhat surprising and in contrast to the behavior of the other magnetic field formulation based on $H_{z}$. Perhaps the ability of the edge element to efficiently enforce the field continuity and jump conditions at interfaces plays a role. It is worth doing a further convergence study to clarify this fact.

\section{Acknowledgement}

This research was partly supported by the Canadian Institute for Photonics Innovations (CIPI).

\section{References}

1. E. Popov and M. Nevière, "Grating theory: new equations in Fourier space leading to fast converging results for TM polarization," J. Opt. Soc. Am. A 17(10), 1773-1784 (2000).

2. G. Bao, D. C. Dobson, and J. A. Cox, "Mathematical studies in rigorous grating theory," J. Opt. Soc. Am. A 12(5), 1029-1042 (1995).

3. T. Delort and D. Maystre, "Finite-element method for gratings," J. Opt. Soc. Am. A 10(12), 2592-2601 (1993).

4. A. Delâge and K. Dossou, "Polarisation dependent loss calculation in echelle gratings using finite element method and Rayleigh expansion," Opt. Quant. Electron. 36(1-3), 223-238 (2004).

5. R. Petit, ed., Electromagnetic theory of gratings, vol. 22 of Topics in Current Physics (Springer-Verlag, New York, 1980).

6. J.-C. Nédélec, "Mixed finite elements in $\mathbf{R}^{3}$," Numer. Math. 35(3), 315-341 (1980).

7. J.-C. Nédélec, "A new family of mixed finite elements in $\mathbf{R}^{3}$," Numer. Math. 50(1), 57-81 (1986).

8. M. Koshiba, S. Maruyama, and K. Hirayama, "A vector finite element method with the high-order mixed-interpolation-type triangular elements for optical waveguiding problems," J. Lightwave Technol. 12(3), 495-502 (1994). 
9. L. Demkowicz and L. Vardapetyan, "Modeling of electromagnetic absorption/scattering problems using hp-adaptive finite elements," Comput. Methods Appl. Mech. Engrg. 152(1-2), 103-124 (1998).

10. K. Dossou and M. Fontaine, "A high order isoparametric finite element method for the computation of waveguide modes," Comput. Methods Appl. Mech. Engrg., 2004, in press.

11. L. Li, J. Chandezon, G. Granet, and J.-P. Plumey, "Rigorous and Efficient GratingAnalysis Method Made Easy for Optical Engineers," Appl. Opt. 38(2), 304-313 (1999).

12. S. Janz, A. Balakrishnan, S. Charbonneau, P. Cheben, M. Cloutier, A. Delage, K. Dossou, L. Erickson, M. Gao, P. Krug, B. Lamontagne, M. Packirisamy, M. Pearson, and D.-X. $\mathrm{Xu}$, "Planar Waveguide Echelle Gratings in Silica-On-Silicon," IEEE Photon. Technol. Lett. 16(2), 503- 505 (2004).

13. D. Chowdhuri, "Design of low-loss and polarization-insensitive reflection grating-based planar demultiplexers," IEEE J. Select. Topics Quantum Electron. 6(2), 233-239 (2000).

14. P. Clemens, G. Heise, R. Marz, H. Michel, A. Reichelt, and H. Schneider, "8-channel optical demultiplexer realized as $\mathrm{SiO} 2 /$ Si flat-field spectrograph," IEEE Photon. Technol. Lett. 6(9), 1109-1111 (1994).

15. J.-J. He, B. Lamontagne, A. Delage, L. Erickson, M. Davies, and E. Koteles, "Monolithic integrated wavelength demultiplexer based on a waveguide Rowland circle grating in InGaAsP/lnP," J. Lightwave Technol. 16(4), 631-638 (1998).

16. E. Loewen, D. Maystre, E. Popov, and L. Tsonev, "Echelles: scalar, electromagnetic, and real-groove properties," Appl. Opt. 34(10), 1707-1727 (1995). 
Table 1. Quadratic element basis functions: $L_{i}^{n}$ is the Lagrange interpolation polynomial of degree $n$ associated to the triangle node numbered $i$. The numbering notation is given in Fig. 2 .

\begin{tabular}{clc}
\hline Nodes & \multicolumn{2}{c}{ Basis functions } \\
\hline 1 & $\mathbf{v}_{1}=L_{1}^{2}\left(\nabla_{t} L_{2}^{1}\right)$ & $\mathbf{v}_{2}=L_{1}^{2}\left(\nabla_{t} L_{3}^{1}\right)$ \\
2 & $\mathbf{v}_{3}=L_{2}^{2}\left(\nabla_{t} L_{3}^{1}\right)$ & $\mathbf{v}_{4}=L_{2}^{2}\left(\nabla_{t} L_{1}^{1}\right)$ \\
3 & $\mathbf{v}_{5}=L_{3}^{2}\left(\nabla_{t} L_{1}^{1}\right)$ & $\mathbf{v}_{6}=L_{3}^{2}\left(\nabla_{t} L_{2}^{1}\right)$ \\
4 & $\mathbf{v}_{7}=L_{4}^{2}\left(\nabla_{t}\left(L_{2}^{1}-L_{1}^{1}\right)\right)$ & $\mathbf{v}_{8}=L_{4}^{2}\left(\nabla_{t} L_{3}^{1}\right)$ \\
5 & $\mathbf{v}_{9}=L_{5}^{2}\left(\nabla_{t}\left(L_{3}^{1}-L_{2}^{1}\right)\right)$ & $\mathbf{v}_{10}=L_{5}^{2}\left(\nabla_{t} L_{1}^{1}\right)$ \\
6 & $\mathbf{v}_{11}=L_{6}^{2}\left(\nabla_{t}\left(L_{1}^{1}-L_{3}^{1}\right)\right)$ & $\mathbf{v}_{12}=L_{6}^{2}\left(\nabla_{t} L_{2}^{1}\right)$ \\
\hline
\end{tabular}


Table 2. Comparison between the diffraction efficiencies of the grating described in section 5.A as computed by the C Method, FMM and the $\mathbf{v}_{t}$ and $v_{z}$ FEM.

\begin{tabular}{lllll}
\hline Order & C Method & FMM & $v_{z}$ FEM & $\mathbf{v}_{t}$ FEM \\
\hline TE polarization & & & & \\
$R_{-2}$ & 0.5132 & 0.5134 & 0.5139 & 0.5141 \\
$R_{-1}$ & 0.1485 & 0.1476 & 0.1480 & 0.1480 \\
$R_{0}$ & 0.1358 & 0.1345 & 0.1350 & 0.1350 \\
$R_{+1}$ & 0.05891 & 0.05810 & 0.05834 & 0.05834 \\
TM polarization & & & & \\
$R_{-2}$ & 0.7004 & 0.4428 & 0.6984 & 0.6996 \\
$R_{-1}$ & 0.02767 & 0.02019 & 0.02834 & 0.02809 \\
$R_{0}$ & 0.02499 & 0.007737 & 0.02500 & 0.02499 \\
$R_{+1}$ & 0.009757 & 0.0002584 & 0.01035 & 0.01011 \\
\hline
\end{tabular}


Table 3. Convergence of the $\mathbf{v}_{t}$ and $v_{z}$ FEM for the grating presented in section 5.A.

\begin{tabular}{|c|c|c|c|c|c|c|}
\hline \multirow[b]{2}{*}{ Number of triangles } & \multicolumn{3}{|c|}{$v_{z} \mathrm{FEM}$} & \multicolumn{3}{|c|}{$\mathbf{v}_{t} \mathrm{FEM}$} \\
\hline & 556 & 2144 & 8402 & 556 & 2144 & 8402 \\
\hline Number of nodes & 1175 & 4417 & 17065 & 1175 & 4417 & 17065 \\
\hline Truncation order ${ }^{a}$ & $P=7$ & $P=15$ & $P=30$ & $P=7$ & $P=15$ & $P=30$ \\
\hline \multicolumn{7}{|l|}{ Order } \\
\hline \multicolumn{7}{|l|}{ TE polarization } \\
\hline$R_{-2}$ & 0.5087 & 0.5133 & 0.5139 & 0.5174 & 0.5145 & 0.5141 \\
\hline$R_{-1}$ & 0.1480 & 0.1479 & 0.1480 & 0.1482 & 0.1481 & 0.1480 \\
\hline$R_{0}$ & 0.1343 & 0.1349 & 0.1350 & 0.1354 & 0.1350 & 0.1350 \\
\hline$R_{+1}$ & 0.05870 & 0.05837 & 0.05834 & 0.05814 & 0.05831 & 0.05834 \\
\hline CPU (sec.) & 0.54 & 1.64 & 19.69 & 2.03 & 9.86 & 130.53 \\
\hline \multicolumn{7}{|l|}{ TM polarization } \\
\hline$R_{-2}$ & 0.6973 & 0.6983 & 0.6984 & 0.7003 & 0.6999 & 0.6996 \\
\hline$R_{-1}$ & 0.03138 & 0.02873 & 0.02834 & 0.02639 & 0.02783 & 0.02809 \\
\hline$R_{0}$ & 0.02680 & 0.02520 & 0.02500 & 0.02437 & 0.02496 & 0.02499 \\
\hline$R_{+1}$ & 0.01239 & 0.01062 & 0.01035 & 0.009111 & 0.009942 & 0.01011 \\
\hline CPU (sec.) & 0.56 & 1.65 & 19.72 & 2.28 & 9.86 & 133.71 \\
\hline
\end{tabular}

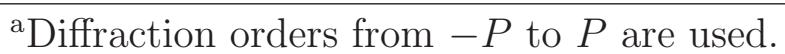


Table 4. Grating with finite conductivity metallic facets: Number of FEM mesh elements (NE), number of nodes (NN) and truncation parameters $P_{1}$ and $P_{2}$.

\begin{tabular}{lllll}
\hline & $\mathrm{NE}$ & $\mathrm{NN}$ & $P_{1}$ & $P_{2}$ \\
\hline$(1)$ & 2763 & 5666 & -32 & 10 \\
$(2)$ & 4806 & 9799 & -36 & 14 \\
$(3)$ & 10919 & 22122 & -36 & 14 \\
$(4)$ & 30313 & 61102 & -82 & 60 \\
\hline
\end{tabular}




\section{List of figure captions}

Fig. 1: Grating profile: $d$ is the grating period.

Fig. 2: The nodes and degrees of freedom of a FEM mesh triangle.

Fig. 3: Grating profile.

Fig. 4: Perfect metallic conductor gratings (order -22): (a) Efficiency curves; (b) PDL curves.

Fig. 5: Grating with only perfectly conductive reflecting facets: (a) Efficiency curves; (b) PDL curves.

Fig. 6: Grating with finite conductivity metallic facets: (a) Efficiency curves; (b) PDL curves.

Fig. 7: Grating with finite conductivity metallic facets: Convergence of the efficiency curves for the TE polarizations ((a) and (b)) and TM polarizations ((c) and (d)). The circles, crosses, diamonds and triangles symbols correspond to the efficiency obtained using respectively the FEM meshes numbered (1), (2), (3) and (4) in Table 4.

Fig. 8: Grating with finite conductivity metallic facets: Convergence of the PDL curves using: (a) the $v_{y}$ formulation; (b) the $\mathbf{v}_{t}$ formulation. The symbol notation is the same as in Fig. 7.

Fig. 9: Finite conductivity metal only on reflecting facets: (a) Efficiency curves; (b) PDL curves. 


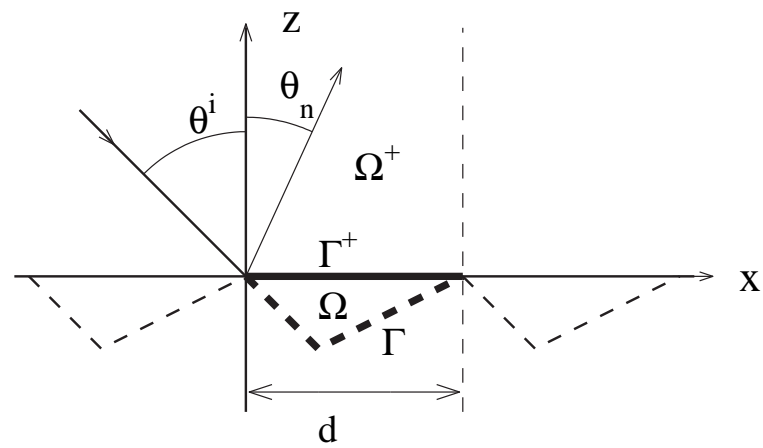

Fig. 1. fig1-54681.eps 


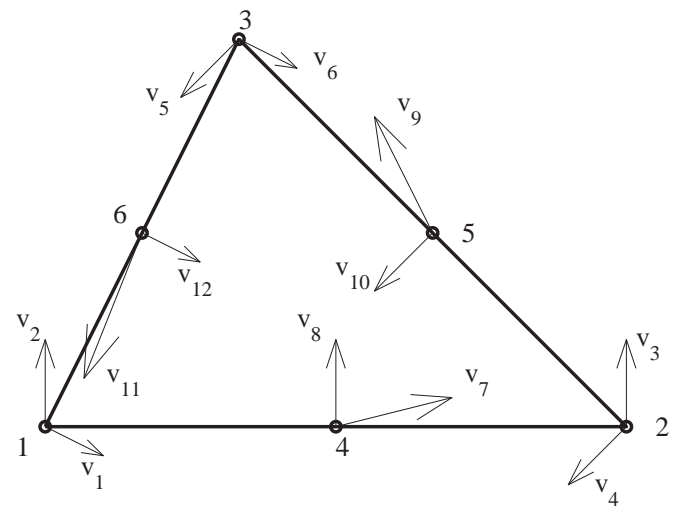

Fig. 2. fig2-54681.eps 


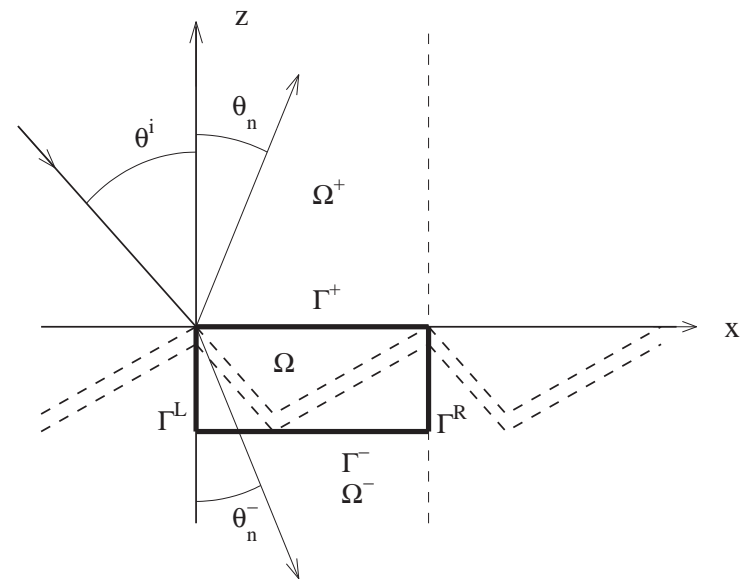

Fig. 3. fig3-54681.eps 
(a)

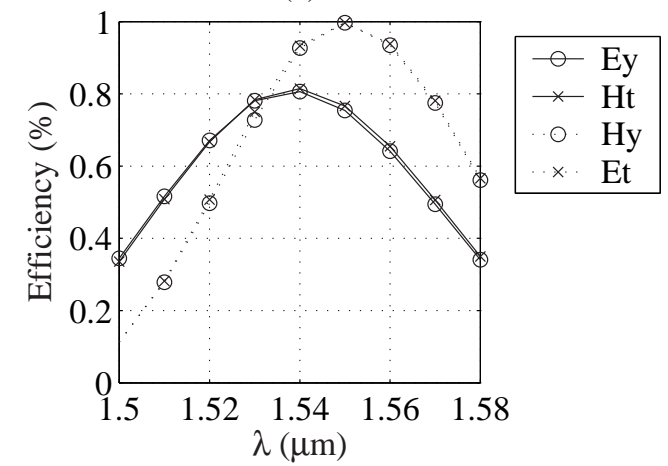

(b)

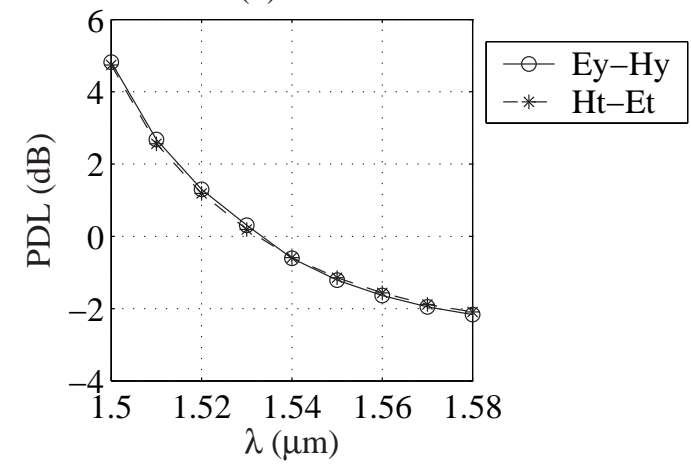

Fig. 4. fig4-54681.eps 
(a)

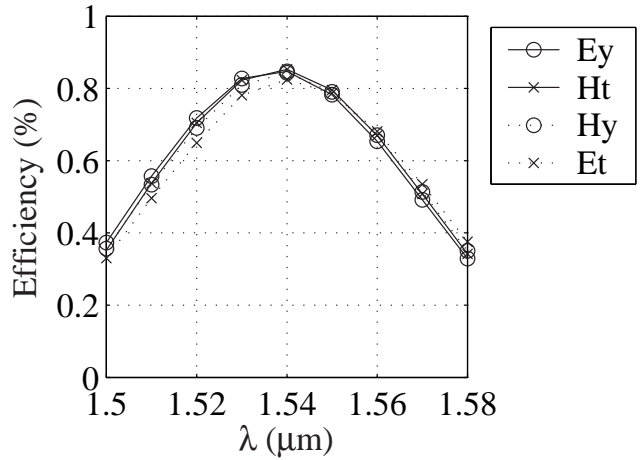

(b)

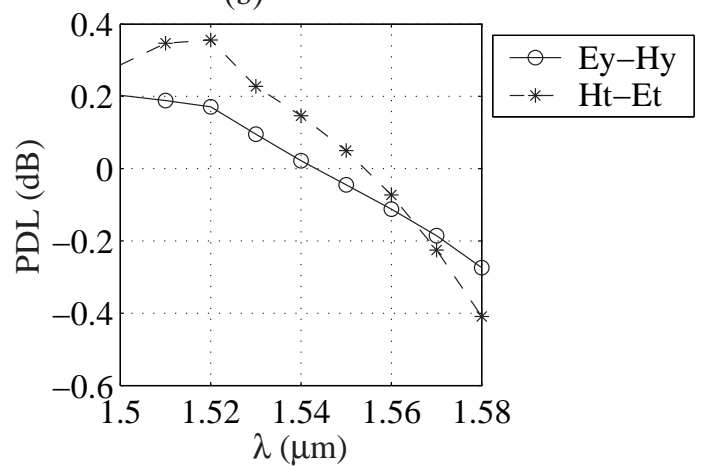

Fig. 5. fig5-54681.eps 
(a)

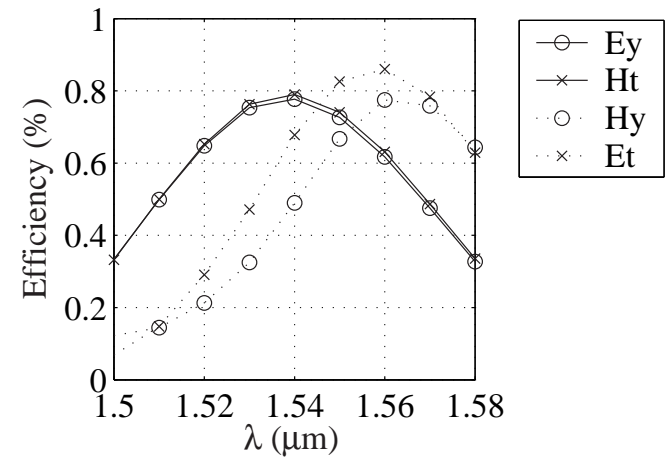

(b)

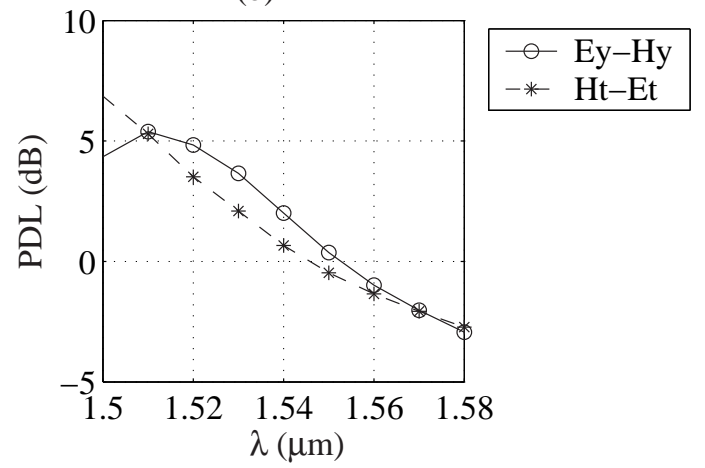

Fig. 6. fig6-54681.eps 
(a)

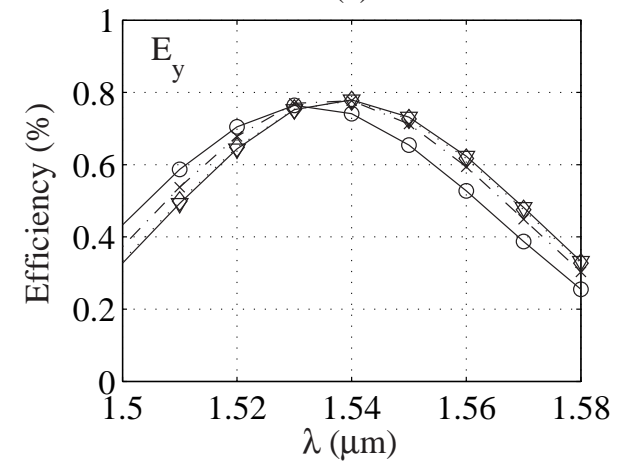

(c)

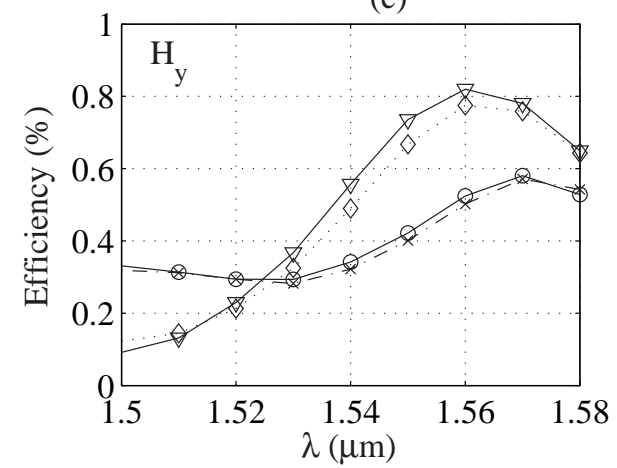

(b)

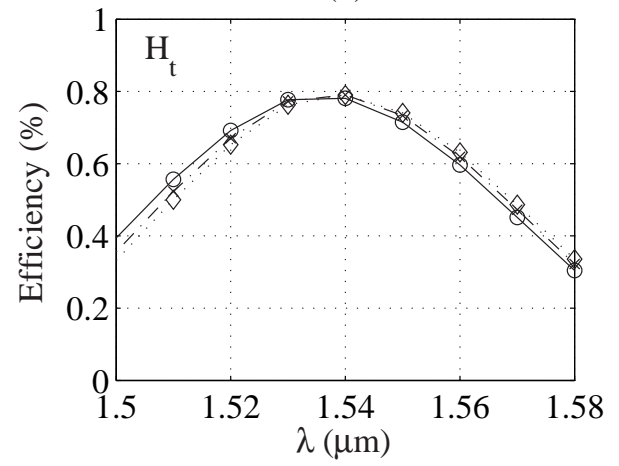

(d)

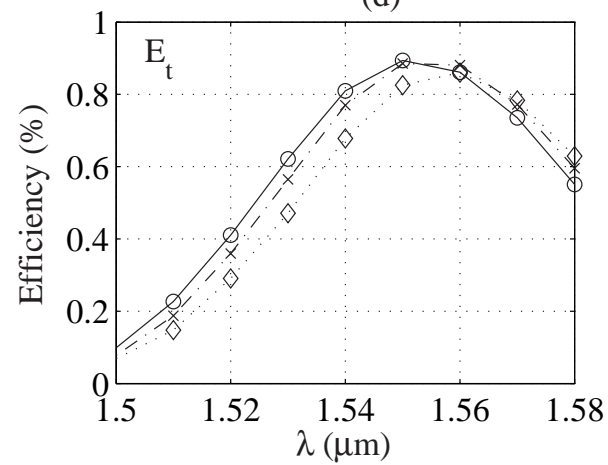

Fig. 7. fig7-54681.eps 

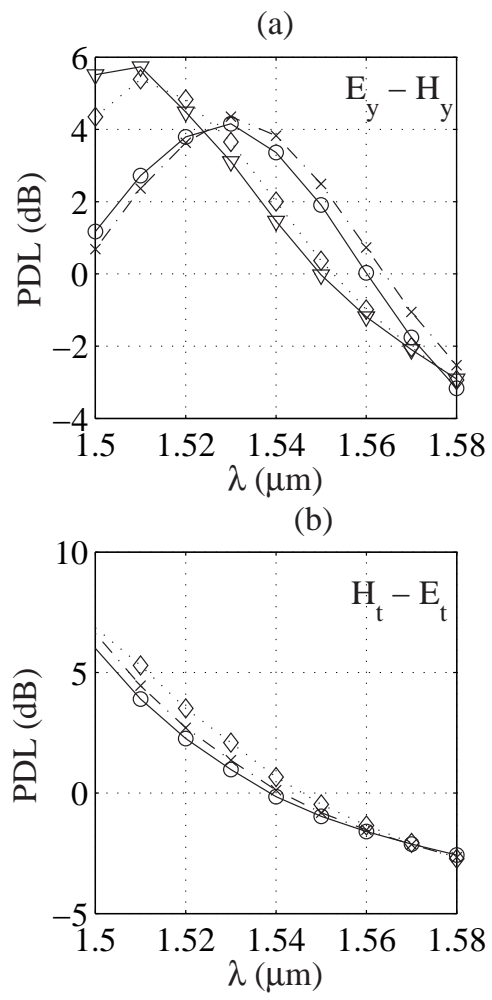

Fig. 8. fig8-54681.eps 
(a)
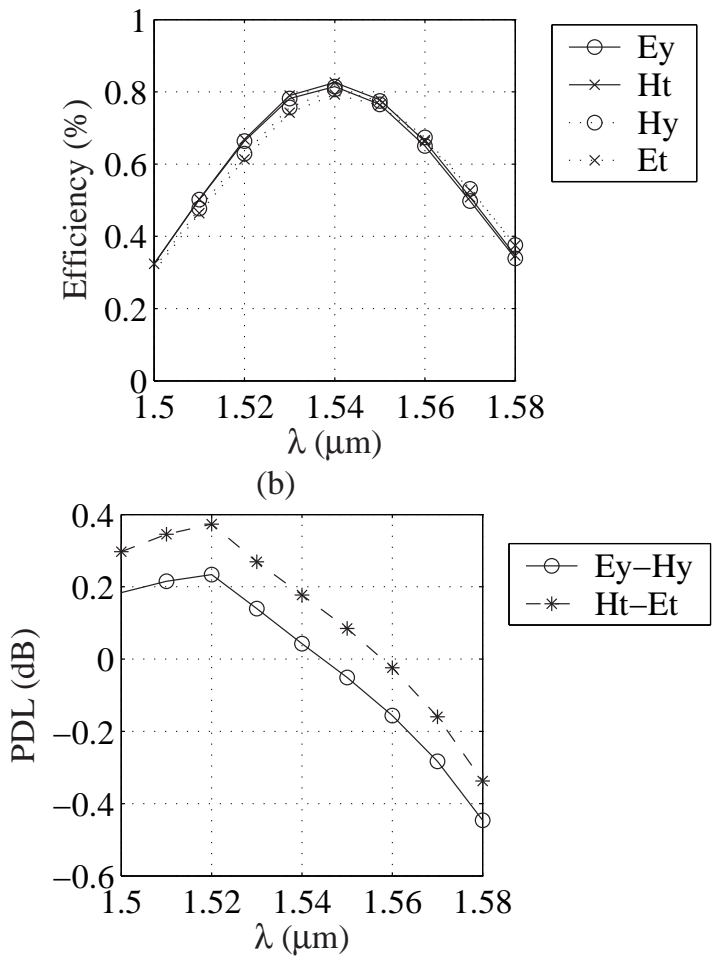

Fig. 9. fig9-54681.eps 